UDC 539.12

DOI: $10.22363 / 2312-9735-2017-25-3-253-265$

\title{
The Boundary Value Problem for Elliptic Equation in the Corner Domain in the Numerical Simulation of Magnetic Systems
}

\author{
E. E. Perepelkin*, R. V. Polyakova ${ }^{\dagger}$, A. D. Kovalenko ${ }^{\dagger}$, P. N. Sysoev*, \\ M. B. Sadovnikova*, A. A. Tarelkin*, I. P. Yudin ${ }^{\dagger}$ \\ * Lomonosov Moscow State University \\ GSP-1, Leninskie Gory, Moscow, 119991, Russian Federation \\ ${ }^{\dagger}$ Joint Institute for Nuclear Research \\ 6 Joliot-Curie St., Dubna, Moscow region, 141980, Russian Federation
}

\begin{abstract}
Modern accelerator systems and detectors contain magnetic systems of complex geometrical configuration. Design and optimization of the magnetic systems demands solving a nonlinear boundary-value problem of magnetostatic.

The region in which the boundary-value problem is solved, consists of two sub-domains: a domain of vacuum and a domain of ferromagnetic. In view of the complex geometrical configuration of magnetic systems, the ferromagnetic/vacuum boundary can be nonsmooth, i.e. it contains a corner point near of which the boundary is formed by two smooth curves crossed in a corner point at some angle.

Thereby, the solution of such a problem has to be found by numerical methods, a question arises about the behavior of the boundary value problem solution around the angular point of the ferromagnetic. This work shows that if the magnetic permeability function meets certain requirements, the corresponding solution of the boundary value problem will have a limited gradient. In this paper, an upper estimate of maximum possible growth of the magnetic field in the corner domain is given.

In terms of this estimate, a method of condensing the differential mesh near the corner domain is proposed. This work represents an algorithm of constructing an adaptive mesh in the domain with a boundary corner point of ferromagnetic taking into account the character of behavior of the solution of the boundary value problem. An example of calculating a model problem in the domain containing a corner point is given.
\end{abstract}

Key words and phrases: magnet systems, mathematical modeling, boundary value problem, elliptic equations, the behavior of solutions in the corner domain

\section{Introduction}

Many physics research facilities use magnetic systems of various configurations, e.g., a system of spectrometric magnets. It is very important to know with a good accuracy the distribution of the magnetic field generated by this system. The problem is actually reduced to formulation of a magnetostatics problem of finding the distribution of the magnetic field generated by the magnetic system under consideration. Since the magnetic system has a complicated configuration, the solution of the problem is usually sought using numerical methods. The domain in which the boundary value problem is solved during calculations of a particular magnetic system often has a piecewise smooth boundary. In this case, the solution of the problem or the derivative solutions can have a singularity. Therefore, the numerical search for the solution requires the use of special methods.

\section{Formulation of the Boundary Value Problem}

The problem to be formulated is the magnetostatics problem of the magnetic field distribution in the corner domain of a ferromagnetic (see Fig. 1). From the Maxwell's

Received $18^{\text {th }}$ January, 2017. 
equations and boundary relations (no currents are supposed to be in the region under consideration) it follows that

$$
\begin{gathered}
\operatorname{div} \vec{B}(p)=0, \quad \operatorname{curl} \vec{H}(p)=0, \quad p \in \Omega ; \\
\vec{n} \cdot\left(\vec{B}_{1}-\vec{B}_{2}\right)=0, \quad \vec{n} \times\left(\vec{H}_{1}-\vec{H}_{2}\right)=0, \quad p \in \Gamma,
\end{gathered}
$$

where $\Omega$ is the ferromagnetic and the vacuum region (on Figs. 1, 2 region $\Omega_{1}=\Omega_{v}$ and region $\Omega_{2}=\Omega_{f}$ ); $\Gamma$ is the boundary; and $\vec{B}$ and $\vec{H}$ are the magnetic field induction and strength vectors, respectively.

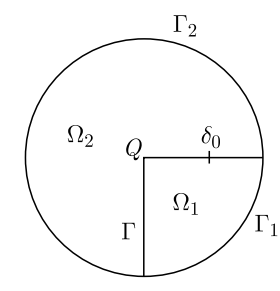

Figure 1. The corner domain

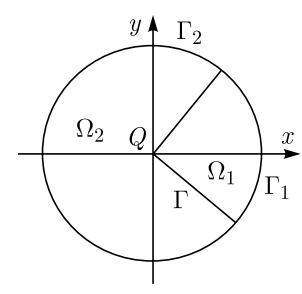

Figure 2. The angular sector

For the ferromagnetic region $\Omega_{2}$, we can write $\vec{B}=\mu_{0} \mu(H) \vec{H}$, where $H=|\vec{H}|, \mu(H)$ is the permeability, and $\mu_{0}$ is the vacuum permeability. For the vacuum region $\Omega_{1}$, we have $\vec{B}=\mu_{0} \vec{H}$. Since there are no current sources in the region $\Omega=\Omega_{1} \cup \Omega_{2}$, the field is potential, and thus the following representation holds:

$$
\vec{H}(p)=-\nabla u(p), \quad p \in \Omega, \quad u(p)= \begin{cases}u_{1}(p), & p \in \Omega_{1}, \\ u_{2}(p), & p \in \Omega_{2},\end{cases}
$$

where $u(p)$ is the scalar potential. The consequent formulation of the boundary value problem is

$$
\begin{gathered}
\operatorname{div}\left[\mu\left(\left|\nabla u_{1}(p)\right|\right) \nabla u_{2}(p)\right]=0, \quad p \in \Omega_{2}, \quad \Delta u_{1}(p)=0, \quad p \in \Omega_{1}, \\
\left.u_{1}\right|_{\Gamma_{-}}=\left.u_{1}\right|_{\Gamma_{+}},\left.\quad \mu\left(\left|\nabla u_{2}(p)\right|\right) \frac{\partial u_{2}}{\partial n}\right|_{\Gamma_{+}}=\left.\frac{\partial u_{1}}{\partial n}\right|_{\Gamma_{-}}, \\
\left.u_{1}\right|_{\Gamma_{1}}=\Psi_{1} ;\left.\quad u_{2}\right|_{\Gamma_{2}}=\Psi_{2},
\end{gathered}
$$

where the function $\mu(H)$ satisfies the conditions:

1. $\mu(H) \in C^{(1)}[0,+\infty)$;

2. $\mu(H)>1$ for $H \in[0,+\infty)$;

3. $\mu(H) \stackrel{H \rightarrow+\infty}{\longrightarrow} 1$.

Let us consider the function $\bar{\mu}(H)$, an analogue of the function $\mu(H)$, for which conditions 2 and 3 are replaced by $\bar{\mu}\left(H^{\prime}\right)=1$ for $H^{\prime} \geqslant H_{0}$, where $H_{0}$ is "large enough". In what follows we will assume that the solution to (1) is $u \in C\left(\Omega \cup \Gamma \cup \Gamma_{1} \cup \Gamma_{2}\right)$, and thus it follows that $\exists C_{0}>0 \forall p \in \Omega \cup \Gamma \cup \Gamma_{1} \cup \Gamma_{2}:|u(p)|<C_{0}$. 


\section{On a Certain Boundary Value Problem}

Before proceeding to the main statements of the paper, we consider an auxiliary problem that is discussed in detail in [1], namely, the boundary value problem (see Fig. 2)

$$
\begin{aligned}
& \left\{\begin{array}{l}
\operatorname{div}[q \nabla u(p)]=0, \quad p \in \Omega, \\
\left.u\right|_{\Gamma_{-}}=\left.u\right|_{\Gamma_{+}}, \\
\left.q_{2} \frac{\partial u}{\partial n}\right|_{\Gamma_{+}}=\left.q_{1} \frac{\partial u}{\partial n}\right|_{\Gamma_{-}}, \quad \text { where } \quad q= \begin{cases}q_{1}, & p \in \Omega_{1}, \\
q_{2}, & p \in \Omega_{2},\end{cases} \\
\left.u\right|_{\Gamma_{1}}=\Psi_{1} ;\left.\quad u\right|_{\Gamma_{2}}=\Psi_{2}, \\
\Omega=\Omega_{1} \cup \Omega_{2}, \quad \Omega_{1}=\left\{(r, \phi): \quad 0<r<r_{0}, \quad|\phi|<\pi / 4\right\}, \\
\quad \Omega_{2}=\left\{(r, \phi): 0<r<r_{0}, \quad|\phi|>\pi / 4\right\},
\end{array}\right.
\end{aligned}
$$

where $\Psi_{i} \in C^{(1)}\left(\Gamma_{i}\right), i=1,2$, (see Fig. 2). Let us introduce the polar coordinate system. The solution is sought by the method of separation of variables: $u \sim R(r) \cdot \Phi(\varphi)$. As a result, we have $\Phi^{\prime \prime}+\lambda^{2} \Phi=0, r^{2} R^{\prime \prime}+r R^{\prime}-\lambda^{2} R=0$.

Thus, by virtue of boundedness of $u(p)$ at the origin, the solution for $R(r)$ will be $R(r) \sim r^{\lambda}$, and for $\Phi(\varphi)$ there will be eigenfunctions divided into two groups, symmetric about $\varphi=0$ and antisymmetric about $\varphi=0$. In the former case, the eigenfunctions take the form

$$
\Phi_{\lambda}^{(1)}(\varphi)= \begin{cases}\cos (\lambda \varphi), & |\varphi|<\pi / 4 \\ a_{\lambda} \cos (\lambda(\pi-\varphi)), & |\varphi|>\pi / 4\end{cases}
$$

Here the constant $a_{\lambda}$ is determined from the boundary ratio for normal derivatives

$$
a_{\lambda}=-\left[q_{1} \sin \left(\lambda \frac{\pi}{4}\right)\right] /\left[q_{2} \sin \left(\lambda \frac{3 \pi}{4}\right)\right],
$$

and the eigenvalues $\lambda$ can be determined using the continuity relation for the solution $u(r, \varphi)$ at the boundary

$$
-\frac{q_{1}}{q_{2}}=\frac{\cos \left(\lambda \frac{\pi}{4}\right)}{\cos \left(\lambda \frac{3 \pi}{4}\right)} \frac{\sin \left(\lambda \frac{3 \pi}{4}\right)}{\sin \left(\lambda \frac{\pi}{4}\right)} .
$$

Thus, either $\operatorname{tg}\left(\lambda \frac{\pi}{4}\right)=0$ and hence $\lambda=4 n$, or $\lambda= \pm \lambda_{1} \pm 4 n$, where $\lambda_{1}$ is the smallest root of the equation

$$
-\frac{q_{1}}{q_{2}}=\left[3-\tan ^{2}\left(\lambda \frac{\pi}{4}\right)\right] /\left[1-3 \tan ^{2}\left(\lambda \frac{\pi}{4}\right)\right] .
$$

The singularity is introduced in the solution by the series term $r^{\lambda_{1}} \Phi_{\lambda_{1}}(\varphi)$ at $\lambda_{1}<1$. From (2) it follows that

$$
\lambda_{1}=1 \quad \Leftrightarrow \quad q_{1}=q_{2}
$$

This means that if $q_{1}=q_{2},|\nabla u(p)|$ will be limited. 


\section{Behavior of the Solution of the Boundary Value Problem}

Let us consider boundary value problem (1) with the permeability function $\bar{\mu}$, region $\Omega$ (see Fig. 1).

Statement 1.

$$
\exists K>0 \quad \forall \delta>0 \quad 0<\rho(p, Q)<\delta:|\nabla u(p)|<K,
$$

where $\rho(p, Q)$ is the distance between the points $p$ and $Q$, and by boundedness on $\Gamma$ is meant boundedness on $\Gamma_{+}$and $\Gamma_{-}$.

Proof. We will prove it by contradiction. Let us assume that it is not true. Then

$$
\forall K>0 \quad \exists \delta>0 \quad 0<\rho(p, Q)<\delta:|\nabla u(p)| \geqslant K .
$$

Let us take $K=\max \left(H_{0}, 4 C_{0} \sqrt{\pi}\right), 0<\delta_{0}<\delta$, then for $p: 0<\rho(p, Q) \leqslant \delta_{0}$ the condition

$$
|\nabla u(p)| \geqslant H_{0} \quad \Rightarrow \quad \bar{\mu}(H)=1 .
$$

should hold.

We introduce a polar coordinate system with the origin at the point $Q$. Let $u(r, \varphi)$ be the solution of our boundary value problem satisfying condition (4). Then on $\Gamma_{ \pm}$it should satisfy the conditions

$$
\left.\frac{\partial u}{\partial \varphi}\left(\delta_{0}, \varphi\right)\right|_{\varphi=0+}=\left.\frac{\partial u}{\partial \varphi}\left(\delta_{0}, \varphi\right)\right|_{\varphi=0-},
$$

and by virtue of continuity of $u\left(\delta_{0}, \varphi\right)$

$$
\left.u\left(\delta_{0}, \varphi\right)\right|_{\varphi=0+}=\left.u\left(\delta_{0}, \varphi\right)\right|_{\varphi=0-}=u\left(\delta_{0}, 0\right)
$$

should hold. It follows from (5) and (6) that

$$
\exists \frac{\partial u}{\partial \varphi}\left(\delta_{0}, 0\right)=\left.\frac{\partial u}{\partial \varphi}\left(\delta_{0}, \varphi\right)\right|_{\varphi=0+}=\left.\frac{\partial u}{\partial \varphi}\left(\delta_{0}, \varphi\right)\right|_{\varphi=0-} .
$$

Thus, putting

$$
\begin{gathered}
\bar{\Psi}_{i}(\phi)=u_{i}\left(\delta_{0}, \phi\right), \quad i=1,2, \quad \bar{\Psi}= \begin{cases}\bar{\Psi}_{1}, & \bar{\Gamma}_{1}, \\
\bar{\Psi}_{2}, & \bar{\Gamma}_{2},\end{cases} \\
\bar{\Gamma}=\left\{\left(\delta_{0}, \phi\right): 0<\phi \leqslant 2 \pi\right\},
\end{gathered}
$$

in the $\delta_{0}$-vicinity of the point $Q$, we obtain the boundary value problem

$$
\begin{array}{cc}
\Delta u_{1}(p)=0, \quad p \in \Omega_{1}, \quad \Delta u_{2}(p)=0, & p \in \Omega_{2}, \\
\left.\frac{\partial u_{2}}{\partial n}\right|_{\Gamma_{+}}=\left.\frac{\partial u_{1}}{\partial n}\right|_{\Gamma_{-}},\left.\quad u_{1}\right|_{\Gamma_{-}}=\left.u_{2}\right|_{\Gamma_{+}},\left.\quad u\right|_{\bar{\Gamma}}=\bar{\Psi},
\end{array}
$$

where $\bar{\Psi} \in C^{(1)}(\bar{\Gamma})$. From (3) (and also from [1]) we find that (7) has no singularities, i.e., $\lim _{p \rightarrow Q}|\nabla u(p)|=\sqrt{a_{1}^{2}+b_{1}^{2}} \leqslant 4 C_{0} \sqrt{\pi}=K$, where $a_{1}$ and $b_{1}$ are the Fourier series coefficients for the function $u(p)$ at the boundary of $\bar{\Gamma}$. Consequently, we have arrived at a contradiction, which proves our statement. 
Thus, it follows from the statement that the magnetic field is bounded in the corner domain provided that the permeability function satisfies the conditions:

1) $\bar{\mu}(H) \in C^{(1)}[0,+\infty)$;

2) $\exists H_{0}>0 \quad \forall H^{\prime} \geqslant H_{0}: \bar{\mu}\left(H^{\prime}\right)=1$.

Note an interesting fact. Let us solve boundary value problem (1) and let its solution have unbounded $|\nabla u|$. This means that in the vicinity of the point $Q$ the permeability function $\mu(|\nabla u|)$ will tend to unity. Since the number of figures in the mantissa is limited, it will turn out that in a certain small vicinity of the point $Q$ the function $\mu(|\nabla u|)$ will be equal to 1 . That is, there arises boundary value problem (1) with the permeability function $\bar{\mu}(H)$ that has bounded $|\nabla u|$ and thus we get a contradiction. Consequently, numerical calculations cannot "theoretically" yield a solution with the infinitely growing $|\nabla u|$, and we will seek the solution of another boundary value problem, namely, problem (1) with $\bar{\mu}(H)$, where $|\nabla u|$ is limited. But the solution of problem (1) with $\bar{\mu}(H)$ does not coincide in the general case with the solution of initial problem $(7)$ with $\mu(H)$. It is therefore necessary to use special methods for solving this problem. One of these methods is considered in $[2,3]$ for the solution of the equation $\operatorname{div}[\mu(|\nabla u|) \nabla u]=0$ in the corner domain.

\section{Estimation of the Magnetic Field Growth}

Let us show that the magnetic field in the corner domain of a ferromagnetic satisfies the condition

$$
H(s) \leqslant C_{0} \ln \frac{1}{r_{s}}+w(s),
$$

where $C_{0}$ is a constant; $w(s)$ is a bounded function; and $r_{s}$ is the distance to the corner. The integral formulation of the magnetostatic problem allows the magnetic field to be represented as

$$
\vec{H}(s)=\vec{H}_{C}(s)-\nabla_{s} \int_{\Omega_{f}}\left(\vec{M}(p), \nabla_{P} \Psi(s, p)\right) \mathrm{d} v_{p},
$$

where $\vec{H}_{C}$ is the field from the current sources; $\vec{M}$ is the ferromagnetic magnetization vector; the function $\Psi(s, p)$ is equal to $\frac{1}{4 \pi r_{\mathrm{sp}}}$ or $\frac{1}{2 \pi} \ln r_{\mathrm{sp}}$ for the three-dimensional and the two-dimensional case, respectively; and $\Omega_{f}$ is the ferromagnetic domain (see Fig. 2: region $\Omega_{1}=\Omega_{v}$ and region $\Omega_{2}=\Omega_{f}$ ). The magnetization vector is defined as $\vec{M}=\mu_{0} \chi(H) \vec{H}=\mu_{0}(\mu(H)-1) \vec{H}$, where $\mu_{0}$ is a constant; $\chi(H)$ is the magnetic susceptibility; and $\mu(H)$ is the permeability of the ferromagnetic. Given high fields $(H \rightarrow \infty)$, the representation $\mu(H)=1+\frac{A}{H}-\frac{B}{H^{2}}, H \rightarrow \infty$ is valid, where $A$ and $B$ are positive constants. Consequently, when $H \rightarrow \infty, M=|\vec{M}|$ is limited by a constant $M_{0}=\mu_{0} A$. Let us consider the $2 \mathrm{D}$ case. From (9) we obtain

$$
\vec{H}(s)=\vec{H}_{C}(s)-\frac{1}{2 \pi} \nabla_{s} \int_{\Omega_{f}}\left(\vec{M}(p), \frac{\vec{r}_{\mathrm{sp}}}{r_{\mathrm{sp}}^{2}}\right) \mathrm{d} v_{p} .
$$

Here the first term is limited, and we therefore estimate the second term 


$$
\left|\nabla_{s} \int_{\Omega_{f}}\left(\vec{M}(p), \frac{\vec{r}_{\mathrm{sp}}}{r_{\mathrm{sp}}^{2}}\right) \mathrm{d} v_{p}\right| \leqslant 2 \int_{\Omega_{f}} \frac{2 r_{\mathrm{sp}}^{2}\left|M^{(x)}\right|+2 r_{\mathrm{sp}}^{2}\left|M^{(y)}\right|}{r_{\mathrm{sp}}^{4}} \mathrm{~d} v_{p} \leqslant 8 M_{0} \int_{\Omega_{f}} \frac{1}{r_{\mathrm{sp}}^{2}} \mathrm{~d} v_{p} .
$$

We calculate the integral

$$
\int_{\Omega_{f}} \frac{1}{r_{\mathrm{sp}}^{2}} \mathrm{~d} v_{p}=\int_{v_{\delta}} \frac{1}{r_{\mathrm{sp}}^{2}} \mathrm{~d} v_{p}+\int_{\Omega_{f} \backslash v_{\delta}} \frac{1}{r_{\mathrm{sp}}^{2}} \mathrm{~d} v_{p}
$$

where $v_{\delta}=\Omega_{f} \cap S_{\delta}(Q)$ is the angular sector at the $v_{\delta}$ corner point $Q$ (see Fig. 2).

The integral over the domain $\Omega_{f} / v_{\delta}$ will be limited, and we therefore consider only the integral over the domain $v_{\delta}$

$$
\begin{aligned}
\int_{\Omega_{f}} \frac{1}{r_{\mathrm{sp}}^{2}} \mathrm{~d} v_{p}= & \int_{0}^{\omega_{0}} \mathrm{~d} \varphi_{p} \int_{0}^{\delta} \frac{r_{p} \mathrm{~d} r_{p}}{r_{p}^{2}+r_{s}^{2}-2 r_{p} r_{s} \cos \varphi_{\mathrm{sp}}}= \\
& =\int_{0}^{\omega_{0}} \mathrm{~d} \varphi_{p}\left(\int_{0}^{1} \frac{t \mathrm{~d} t}{1+t^{2}-2 t \cos \varphi_{\mathrm{sp}}}+\int_{1}^{\delta / r_{s}} \frac{t \mathrm{~d} t}{1+t^{2}-2 t \cos \varphi_{\mathrm{sp}}}\right), \quad t=\frac{r_{p}}{r_{s}} .
\end{aligned}
$$

Then we use the expression for the generating function

$$
\frac{1}{\sqrt{1+t^{2}-2 t \cos \varphi_{\mathrm{sp}}}}= \begin{cases}\sum_{m=0}^{+\infty} P_{m}\left(\cos \varphi_{\mathrm{sp}}\right) t^{m}, & |t|<1, \\ \sum_{m=0}^{+\infty} P_{m}\left(\cos \varphi_{\mathrm{sp}}\right) t^{-m-1}, & |t|<1,\end{cases}
$$

and obtain

$$
\begin{array}{r}
\int_{\Omega_{f}} \frac{1}{r_{\mathrm{sp}}^{2}} \mathrm{~d} v_{p}=\omega_{0} \ln \frac{\delta}{r_{s}}+\sum_{m, k=0}^{+\infty} P_{m} P_{k} \frac{\alpha_{m, k}}{m+k+2}+\sum_{m+k \neq 0}^{+\infty} P_{m} P_{k} \frac{\alpha_{m, k}}{m+k}\left(\left(\frac{r_{s}}{\delta}\right)^{m+k}-1\right)= \\
=C_{1} \ln \frac{1}{r_{s}}+w_{1}(s),
\end{array}
$$

where

$$
\alpha_{m, k}=\int_{0}^{\omega_{0}} P_{m}\left(\cos \varphi_{\mathrm{sp}}\right) P_{k}\left(\cos \varphi_{\mathrm{sp}}\right) \mathrm{d} \varphi_{p},
$$

$C_{1}$ is a constant, and $w_{1}(s)$ is a bounded function. Thus, the validity of expression (8) is ascertained.

\section{Method of Mesh Condensing in the Corner Domain}

In $[4,5]$ there are examples of constructing a differential mesh for some boundary value problems in corner domains. The main idea is to condense the differential mesh or finite 
elements for obtaining an admissible problem approximation error. This error involves integrals over elementary domains estimated by the quantities of the form $C h_{i}^{\beta}\|u\|_{k, j}$, where $h_{i}$ is the diameter of the $i$ elementary domain or mesh cell; $\beta$ is a positive number; $\|u\|_{k, j}$ is the norm of the function with the $k$ th derivative in this domain; and $C$ is a constant independent of all these factors. Then we can require, for example, that quantities $C h_{i}^{\beta}\|u\|_{k, j}$ be identical in the domain under consideration. To this end, $h_{i}^{\beta}$ can be decreased in inverse proportion to $\|u\|_{k, j}$ on approach to the singular points. We demonstrate the validity of the following statement.

Let $\vec{V}(s)$ be the solution of the magnetostatic problem in the integral formulation found by a numerical method and $\vec{H}(s)$ be the exact solution. Then the following estimate is valid:

$$
\|\vec{V}-\vec{H}\|_{L_{2}(D)}^{2}<h^{2}\left(c_{1} \ln ^{2} h+c_{2} \ln h+c_{3}\right),
$$

where $c_{1}, c_{2}$, and $c_{3}$ are positive constants and $h$ is the diameter of the domain $D$, which is a differential grid cell containing the ferromagnetic corner.

By virtue of (9), the following expression for $\vec{V}(s)$ holds:

$$
\vec{V}(s)=\vec{H}_{C}(s)-\frac{1}{2 \pi} \nabla_{s} \sum_{j=1}^{N} \int_{\Omega_{j}}\left(\vec{M}\left(\vec{H}_{j}\right), \frac{\vec{r}_{s p_{j}}}{r_{s p_{j}}^{2}}\right) \mathrm{d} v
$$

where $\vec{H}_{j}$ is the field in the cell $\Omega_{j}, j=1 \ldots N ; \bigcup_{j=1}^{N} \Omega_{j}=\Omega_{f}$; and $r_{s p_{j}}$ is the distance from the point $s$ to the point $p_{j} \in \Omega_{j}$. We consider the difference

$$
\vec{V}(s)-\vec{H}(s)=-\frac{1}{2 \pi} \nabla_{s} \sum_{j=1}^{N} \int_{\Omega_{j}}\left(\vec{M}\left(\vec{H}_{j}\right)-\vec{M}\left(\vec{H}\left(p_{j}\right)\right), \frac{\vec{r}_{s p_{j}}}{r_{s p_{j}}^{2}}\right) \mathrm{d} v .
$$

Since the quantity $|\vec{M}|<M_{0}$ is limited, it follows that $\left|\vec{M}\left(\vec{H}_{j}\right)-\vec{M}\left(\vec{H}\left(p_{j}\right)\right)\right|<$ $2 M_{0}$ for $j=1 \ldots N$. Thus, we obtain

$$
|\vec{V}(s)-\vec{H}(s)|<\frac{8 M_{0}}{\pi} \sum_{j=1}^{N} \int_{\Omega_{j}} \frac{\mathrm{d} v}{r_{s p_{j}}^{2}}=\frac{8 M_{0}}{\pi} \sum_{j=1}^{N} \int_{\Omega_{j} \cap S_{\delta}(Q)} \frac{\mathrm{d} v}{r_{s p_{j}}^{2}}+\frac{8 M_{0}}{\pi} \sum_{j=1}^{N} \int_{\Omega_{j} \backslash S_{\delta}(Q)} \frac{\mathrm{d} v}{r_{s p_{j}}^{2}} .
$$

As a result, using the estimate obtained above, we arrive at the expression

$$
|\vec{V}(s)-\vec{H}(s)|<C_{2} \ln \frac{1}{r_{s}}+w_{2}(s) .
$$

It remains to estimate $\|\vec{V}-\vec{H}\|_{L_{2}(D)}^{2}$, where the domain $D$ is the $S_{\delta}(Q)-\delta$-domain of the corner point $Q$. Using (12), we obtain

$$
\|\vec{V}-\vec{H}\|_{L_{2}(D)}^{2}=\int_{D}|\vec{V}(s)-\vec{H}(s)|^{2} \mathrm{~d} v<h^{2}\left(c_{1} \ln ^{2} h+c_{2} \ln h+c_{3}\right),
$$


where $h=2 \delta$, and $c_{1}, c_{2}$, and $c_{3}$ are constants. The result corresponds to (10). Based on the aforesaid and inequality (10), we propose a differential mesh condensing method

$$
\int_{0}^{h_{1}}\left|\ln \frac{1}{x}\right| \mathrm{d} x=d_{0}, \quad \int_{x_{m-1}}^{x_{m}}\left|\ln \frac{1}{x}\right|^{2} \mathrm{~d} x=d_{0}, \quad x_{m}-x_{m-1}=h_{m}, \quad m=1,2 \ldots, M .
$$

Here $d_{0}$ is a constant; $M$ is the number of partitions along the coordinate axis $(O X$ or $O Y$ ) in the corner domain; $h_{m}$ is the grid spacing; and $x_{m}$ is the coordinate of the grid node along the $O X$ or $O Y$ axis (the origin of the coordinates is at the corner point), $|x|<1$.

\section{Calculation of the Magnetic Field in the Corner Domain}

In Section 5 we gave the upper estimate of the admissible magnetic field growth in the corner domain of a ferromagnetic. In Section 6, based on this estimate, we proposed a method of condensing the differential mesh in the corner domain. In this section we present numerical calculations of a magnetic system using this method. It is evident from the calculations that the above mesh condensing method substantially improves the accuracy of the calculated magnetic field distribution. We considered a magnetic system depicted in Fig. 2. The domain $\Omega_{v}$ corresponds to the vacuum, and the domain $\Omega_{f}$ is filled with a ferromagnetic. A boundary value problem

$$
\left\{\begin{array}{l}
\frac{\partial}{\partial x} v \frac{\partial A_{z}}{\partial x}+\frac{\partial}{\partial y} v \frac{\partial A_{z}}{\partial y}=0, \quad p \in \Omega_{f} \cup \Omega_{v}=\Omega \\
\left.A_{z}\right|_{\Gamma_{-}}=\left.A_{z}\right|_{\Gamma_{+}},\left.\quad v \frac{\partial A_{z}}{\partial n}\right|_{\Gamma_{+}}=\left.\frac{\partial A_{z}}{\partial n}\right|_{\Gamma_{-}}, \\
\left.A_{z}\right|_{x=0}=C,\left.\quad A_{z}\right|_{x=x_{2}}=-C,\left.\quad \frac{\partial A_{z}}{\partial n}\right|_{y=0, y_{1}}=0
\end{array}\right.
$$

corresponding to the formulation of the magnetostatic problem with respect to the vector potential $A_{z}$, was solved in the domain under consideration. The function $\vartheta$ is defined as $\vartheta=1 / \mu(B)$, where $\mu(B)$ is the permeability of the ferromagnetic; $B$ is the modulus of the magnetic induction vector $\vec{B}=\operatorname{rot} \vec{A} ; \Gamma_{ \pm}$is the interface; and $C$ is a constant. The efficiency of the differential mesh condensing method described in Section 6 was estimated by the following calculations:

Variant 1. The solution to problem (13) was found on a sequence of $(10 k) \times(10 k)$ meshes, where $k=1,2,4,8,10,20,40$; that is, $10 \times 10,20 \times 20, \ldots, 400 \times 400$ meshes were obtained. The mesh spacing in the corner domain $\Pi_{Q}$ was uniform.

Variant 2. Problem (13) was calculated on the same sequence of meshes at $k=$ $1,2,4,8$, but the mesh spacing in the domain $\Pi_{Q}$ was chosen using the differential mesh condensing method described in Section 6. In the domain $\Omega / \Pi_{Q}$ the mesh spacing was not changed as compared with the previous variant. The number of node points in the domain $\Pi_{Q}$ was the same, and only their distribution was changed.

The results of the variant 1 calculations were taken to be a sort of reference because the accuracy of the calculated solution was assumed to increase with increasing number of partitions, except probably for a particular corner domain. Then the results of the variant 2 calculations were compared with the results of the variant 1 calculations. Figures 3-6 shows distributions $B_{y}(x)$ at $y=y_{1}$ for different meshes. In all figures the reference distribution $B_{y}(x)$ calculated on the $400 \times 400$ mesh is designated as trace 1 . 
The plots of traces 2 and 3 are the distributions $B_{y}(x)$ calculated by variants 1 and 2 , respectively. The distributions in Figs. $3-6$ are calculated on the $10 \times 10,20 \times 20,400 \times 400$, and $80 \times 80$ meshes, respectively. It is evident from Figs. 3 that the accuracy of the variant 2 calculations (nonuniform mesh) is substantially higher than that of the variant 1 calculations (uniform mesh). Thus, it follows that the proposed method of constructing a differential mesh in the corner domain is worth using and yields results comparable in accuracy with the results obtained only on meshes with the number of nodes in each axis four to five times greater than in the initial mesh.An algorithm of thickening differential mesh near the corner point has been developed. It allows one to significantly reduce the computation time and simultaneously to increase the accuracy of the solution of the boundary value problem. Variant 1 - maximum of relative error is $11.085 \%$; variant 2 - maximum of relative error is $1.091 \%$.

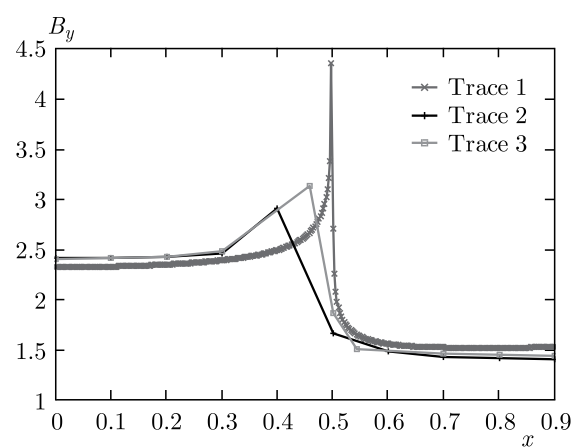

Figure 3. Distributions $B_{y}(x)$ at $y=y_{1}$. Mesh 10x10

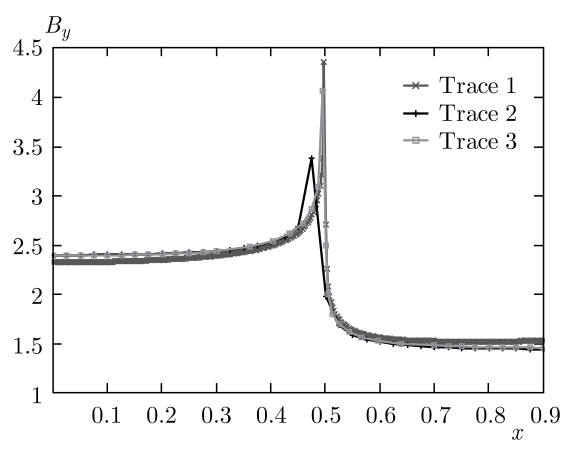

Figure 5. Distributions $B_{y}(x)$ at $y=y_{1}$. Mesh 400x400

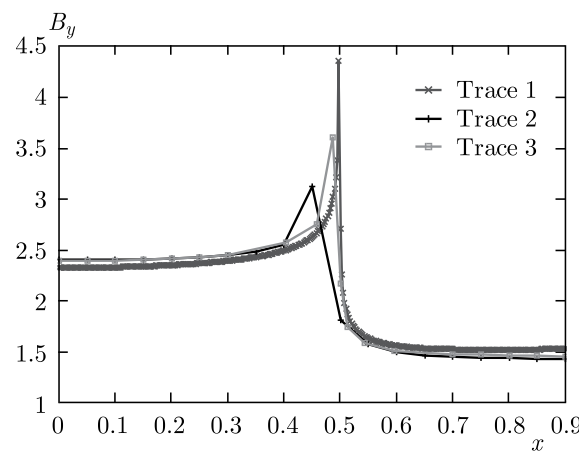

Figure 4. Distributions $B_{y}(x)$ at $y=y_{1}$. Mesh 20x20

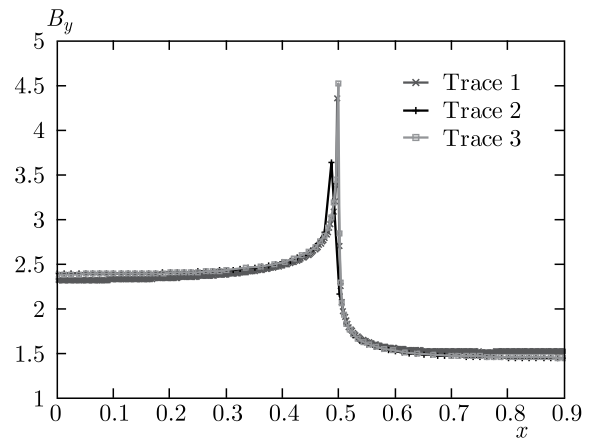

Figure 6. Distributions $B_{y}(x)$ at $y=y_{1}$. Mesh 80x80

\section{Results of Modeling of Some Magnetic Systems}

The significance of numerical modeling at the investigating of magnetic systems is defined by not only known dignities of computational experiment but also that the measurement of magnetic field is labor-intensive and expensive problem. The process of the mathematical modeling of magnetic systems (see Figs. 7 and 8), as the authors of this work have presented, should be divided into two large stages. 


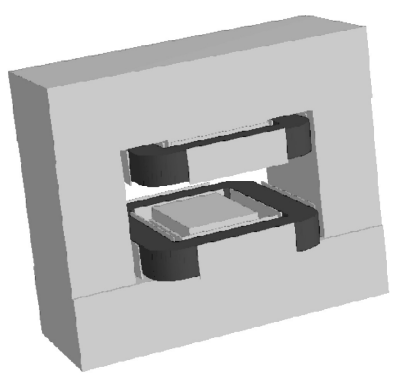

Figure 7. General view of spectrometric magnet $1 \mathrm{SP}-40-4 \mathrm{~B}$

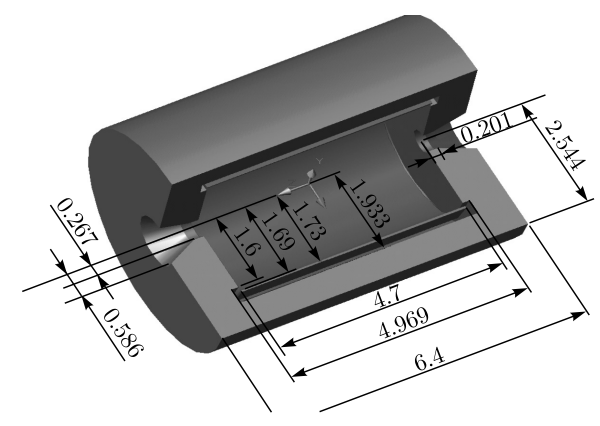

Figure 8. Type of solenoidal magnet

\subsection{Results of modeling magnetic systems SP-94 and 1SP-40-4B}

In the experiment performed at the Veksler and Baldin Laboratory of High Energy Physics, JINR, the SP-94 [6] magnet is used. It was necessary to select the configuration of the core and the current coils such that the quantity $\int_{-\infty}^{+\infty} B_{y}(0,0, z) \mathrm{d} z$ had the maximum value. Fig. 9a presents the distribution of $B_{y}$ in the $X O Z$ plane for this configuration of the magnet $S P=94$ (variant 1). First, the initial configuration (variant 1) was calculated. Here $\int_{-L}^{L} B_{y}(0,0, z) \mathrm{d} z=2.314$, where $L=1.5 \mathrm{~m}$ is the dimension along the $O Z$ axis of the region where the magnetic field was calculated for variant 1 . Second, the initial configuration (variant 2) was calculated. Fig. 9b presents the distribution of $B_{y}$ in the $X O Z$ plane for configuration of the magnet $S P=94$ (variant 2). Here $\int_{-L}^{L} B_{y}(0,0, z) \mathrm{d} z=2.987$, which is 1.291 times greater than for the initial configuration in variant 1 .

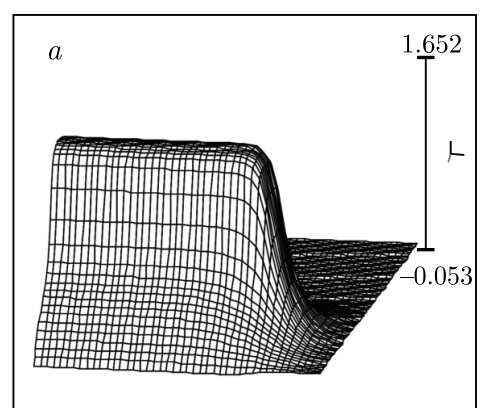

BY0

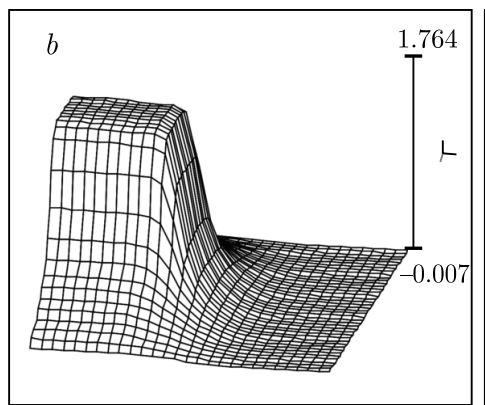

BY0

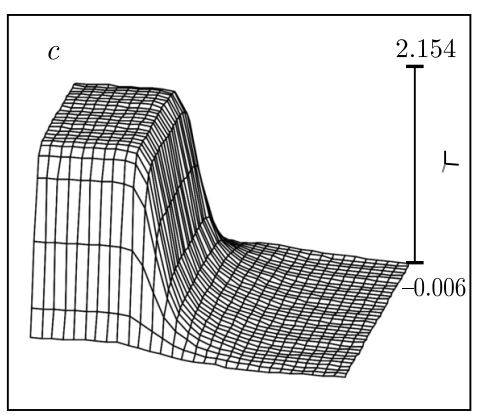

BY0

Figure 9. Distribution of $B_{y}$ for three configurations of the magnet SP-94

Two configurations of the magnet 1SP-40-4B NIS (variant 1, 2, respectively) for which the numerical computations of the magnetic fields were performed, i.e., in fact, a nonlinear inverse problem of magnetostatics were solved. The purpose of the simulation is to find by a calculation method such a magnet geometry that the region of the homogeneity of the magnetic field would be essentially larger as compared to the existing magnet 
configuration. Fig. 10 present distributions of the components of the magnetic field for our two variants of configurations of the magnet $1 \mathrm{SP}-40-4 \mathrm{~B}$ in a $3 \mathrm{D}$ case. Clearly, for variant 2 the distributions of the components got more smooth.
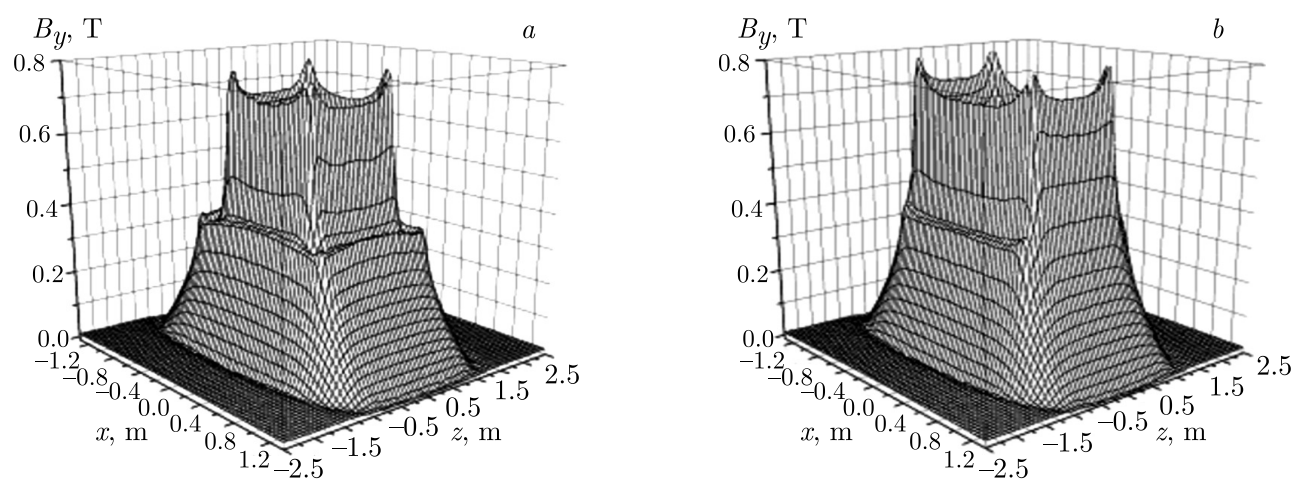

Figure 10. Dependences $B_{y}(x, 0.3, z)$ for two configurations of the magnet, current 1100 A

\subsection{The Solenoid Type Magnetic Field Detector Modeling}

Magnetic systems are very important parts $[7,8]$. To create the necessary configuration of magnetic field, the repeated solution of nonlinear boundary value problem of magnetostatics is needed. In the present work, we consider the problem of creation of homogeneous map of magnetic system of solenoidal type (see Fig. 8). As a result of optimization, the geometric parameters of magnetic system were chosen in such a way so as to get maximal size of the domain of homogeneity of the magnetic field.

Due to symmetry, only $1 / 24$ part of the geometry with corresponding boundary conditions is modeled. The calculations were performed (using two software products: TOSCA and native MFC) by the method of finite elements on tetrahedral mesh with 5000000 elements.

In Figs. 11 and 12 the domains with the degrees of homogeneity of magnetic field are of $0.1 \%$ and $0.5 \%$, respectively. The black continuous line shows that the homogeneity of $0.1 \%$ is needed. In Fig. 11, the scale of magnetic field has site from $0.99-1.001 \mathrm{~T}$, in Fig. 12 from $0.998-1.002 \mathrm{~T}$.
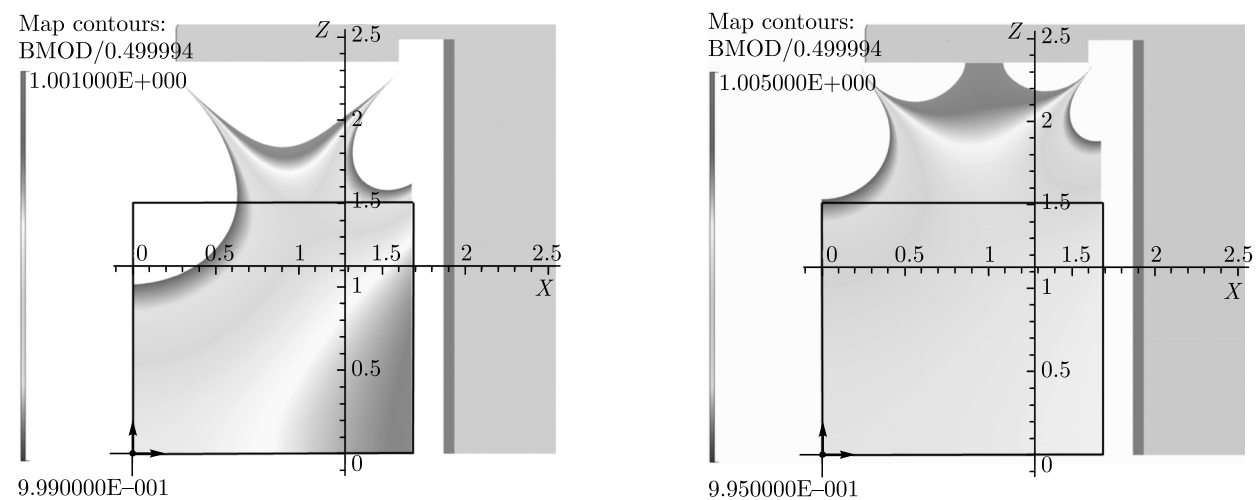

Figure 11. Field homogeneity is $\pm 0.1 \%$ Figure 12. Field homogeneity is $\pm 0.5 \%$ 


\section{Conclusions}

1. The upper estimate for the admissible growth of the magnetic field $\vec{H}(p)$ in the corner domain

$$
H(p) \leqslant C_{0} \ln \frac{1}{r_{p}}+w(p),
$$

where $C_{0}$ is a constant, $w(p)$ is a bounded function, and $r_{p}$ is the distance to the corner, is asymptotically obtained for the case of $\mu(H) \rightarrow 1$ when $H \rightarrow \infty$.

2. A method of condensing the differential mesh in the corner domain is proposed, which appreciably improves the accuracy of the calculated solution.

3. The numerical modeling results are presented for the SP-94 magnet system in the Delta-Sigma experiment performed within the Topical Plan of JINR on international collaboration. Two-dimensional and three-dimensional modeling of the configuration of the magnet core and current coils was performed to obtain the maximum value of the integral

$$
\int_{-L}^{L} B_{y}(0,0, z) \mathrm{d} z .
$$

4. By a numerical method a configuration of the magnet 1SP-40-4B VBLHEP, JINR has been selected for which the width of the domain of the homogeneity of the magnetic field has grown up from $0.5 \mathrm{~m}$ to $1.0 \mathrm{~m}$, i.e., twice. This growth considerably increases the accuracy of regenerating the pulses of decay particles in the physical reaction under study (search for pentaquarks).

5. As a result of optimization, the geometric parameters of the solenoid type magnetic field detector were chosen in such a way so as to get maximal size of the domain of homogeneity of the magnetic field.

\section{References}

1. G. Strang, G. Fix, An Analysis of the Finite Element Method. Second edition, Wellesley-Cambridge Press, 2008.

2. E. P. Zhidkov, E. E. Perepelkin, An analytical approach for Quasi-Linear Equation in Secondary Order, Computational Methods in Applied Mathematics 1 (2001) 285-297.

3. E. E. Perepelkin, R. V. Polyakova, I. P. Yudin, The Boundary Value Problem for Elliptic Equation in the Corner Domain, Bulletin of Peoples' Friendship University of Russia (2) (2014) 410-414, in Russian.

4. E. A. Volkov, Method of meshes and infinite domains with a piecewise smooth boundary, Dokl. Akad. Nauk SSSR 168(3) (1966) 978-981, in Russian.

5. V. V. Shaidurov, Numerical Solution of the Dirichlet Problem in a Domain with Angles, Nauka, Novosobirsk, 1982, in Russian.

6. I. P. Yudin, V. A. Panacik, E. E. Perepelkin, R. V. Polyakova, A. N. Petersky, Peculiar Features of Numerical Modeling of the Modified Spectrometer Magnet Field, Computer Research and Modeling 7 (1) (2015) 93-105.

7. E. Perepelkin, et al., The ATLAS Experiment at the CERN Large Hadron Collider, Vol. 3, Aad, JINST, 2008.

8. E. Perepelkin, et al., Commissioning of the Magnetic Field in the ATLAS Spectrometer, Vol. 177-178, 2008. 
УДК 539.12

DOI: $10.22363 / 2312-9735-2017-25-3-253-265$

Краевая задача для уравнения эллиптического типа в области
с углом в математическом моделировании магнитных систем

Е. Е. Перепелкин ${ }^{*}$, Р. В. Полякова ${ }^{\dagger}$, А. Д. Коваленко ${ }^{\dagger}$, П. Н. Сысоев*, М. Б. Садовникова*, А.А. Тарелкин*, И. П. Юдин ${ }^{\dagger}$

* ФГОУ ВПО МГУ им. М.В. Ломоносова

Ленинские горы, д. 1, Москва, Россия, 119991

† Объединённый институт ядерных исследований

ул. Жолио-Кюри, д. 6, г. Дубна, Московская область, Россия, 141980

Современные ускорительные системы и детекторы содержат магнитные системы сложной геометрической конфигурации. Проектирование и оптимизация магнитных систем требует решения нелинейной краевой задачи магнитостатики.

Область, в которой решается краевая задача, состоит из двух подобластей: область вакуума и область ферромагнетика. Из-за сложной геометрической конфигурации магнитных систем граница раздела сред ферромагнетик/вакуум может являться негладкой, то есть содержать угловую точку, в окрестности которой граница образована двумя гладкими кривыми, пересекающимися в угловой точке под некоторым углом.

В связи с тем, что решение краевой задачи приходится искать численными методами, встает вопрос о поведении решения в окрестности угловой точки ферромагнетика. Показано, что если функция магнитной проницаемости удовлетворяет определенным условиям, то соответствующее решение краевой задачи будет иметь ограниченный градиент.

Дается верхняя оценка допустимого роста магнитного поля в окрестности угловой точки. На основании полученной оценки предлагается метод сгущения разностной сетки вблизи угловой точки, учитывающий характер поведения решения краевой задачи. Приводятся примеры расчета магнитных систем в области, содержащей «угловую точку».

Ключевые слова: магнитные системы, математическое моделирование, краевая задача, эллиптические уравнения, поведение решения в угловой точке

\section{Литература}

1. Strang G., Fix G. An Analysis of the Finite Element Method. Second edition. Wellesley-Cambridge Press, 2008.

2. Zhidkov E. P., Perepelkin E. E. An analytical approach for Quasi-Linear Equation in Secondary Order // Computational Methods in Applied Mathematics. - 2001. Vol. 1, issue 3. - Pp. 285-297.

3. Перепёлкин Е. Е., Полякова Р. В., Юдин И. П. Краевая задача для эллиптических уравнений в области с «угловой точкой»// Вестник РУДН. Серия: Математика. Информатика. Физика. - 2014. - Т. 2. - С. 410-414.

4. Волков E. А. Метод сеток и бесконечных доменов с кусочно-гладкой границей // Доклалы академии наук СССР. - 1966. - Т. 168(3). - С. 978-981.

5. Шайдуров В. В. Численное решение задачи Дирихле в области с углами // Вычислительные методы в прикладной математике. - Новосибирск: Наука, 1982.

6. Peculiar Features of Numerical Modeling of the Modified Spectrometer Magnet Field / I. P. Yudin, V. A. Panacik, E. E. Perepelkin, R. V. Polyakova, A. N. Petersky // Computer Research and Modeling. - 2015. - Vol. 7, No 1. - Pp. 93-105.

7. Perepelkin E. et al. The ATLAS Experiment at the CERN Large Hadron Collider. Aad, JINST, 2008. — Vol. 3, 437 p.

8. Perepelkin E. et al. Commissioning of the Magnetic Field in the ATLAS Spectrometer. - 2008. - Vol. 177-178, Pp. 265-266.

(C) Perepelkin E. E., Polyakova R. V., Kovalenko A.D., Sysoev P. N., Sadovnikova M. B., Tarelkin A. A., Yudin I. P., 2017 